University of Warwick institutional repository: http://go.warwick.ac.uk/wrap This paper is made available online in accordance with publisher policies. Please scroll down to view the document itself. Please refer to the repository record for this item and our policy information available from the repository home page for further information.

To see the final version of this paper please visit the publisher's website. Access to the published version may require a subscription.

Author(s): Francis Bloch and Bhaskar Dutta

Article Title: Communication networks with endogenous link strength Year of publication: 2009

Link to published version: http://dx.doi.org/10.1016/j.geb.2008.03.007

Publisher statement: None 


\title{
Communication Networks with Endogenous Link Strength*
}

\author{
Francis Bloch, Bhaskar Dutta ${ }^{\dagger}$
}

February 14, 2008

\begin{abstract}
This paper analyzes the formation of networks when players choose how much to invest in each relationship. We suppose that players have a fixed endowment that they can allocate across links, and in the baseline model, suppose that link strength is an additively separable and convex function of individual investments, and that agents use the path which maximizes the product of link strengths. We show that both the stable and efficient network architectures are stars. However, the investments of the hub may differ in stable and efficient networks. Under alternative assumptions on the investment technology and the reliability measure, other network architectures can emerge as efficient and stable.
\end{abstract}

JEL Classification Numbers: D85, Cro

Keywords: communication networks, network reliability, endogenous link strength.

*We thank two anonymous referees and the associate editor for their perceptive comments. We have also benefited from discussions with C. Blackorby, S. Goyal and M. Jackson and from the comments of participants in seminars and conferences at Brescia, CORE, Guanajuato, University of Maryland at College Park, Montpellier, Montreal, Paris and Saint-Etienne.

†Bloch is at GREQAM, Universite d'Aix-Marseille, 2 rue de Charite, 13002 Marseille, France. He is also affiliated with the University of Warwick. Dutta is in the Department of Economics, University of Warwick, Coventry CV4 7AL, England. 


\section{Introduction}

Following a long tradition in sociology, economists have recently focussed attention on the role of social networks in economic activities. One of the main contributions of this emerging literature has been to propose strategic models of network formation, where self-interested agents establish bilateral links in order to maximize their utility. Following the pioneering work of Bala and Goyal (2000) and Jackson and Wolinsky (1996), most of the literature assumes that agents make a discrete decision - namely, choose whether or not to invest in a link of fixed quality. However, in a wide variety of contexts arising in both formal and informal networks, agents do not only choose with whom they link, but also how much they spend on every link they form. In this paper, our objective is to study a model of network formation, where the quality of links is endogenously chosen by the agents. ${ }^{1}$

Our analysis is centered around communication networks - networks where agents derive positive benefits from the agents with whom they are connected, with benefits decreasing as the distance increases between two agents. Communication networks can either be formal networks (like the phone or internet), or informal networks of social relations. ${ }^{2}$ In formal communication networks, the reliability of a communication link depends on the physical characteristics of the connection; in informal social networks, the strength of a social link depends on the frequency and the length of social interactions. In both cases, it is commonly observed that different links may have different quality, and that agents can choose the amount they invest on every relation. Communication networks thus represent an obvious testing ground for a theory of network formation with endogenous link strength.

The modeling of network formation with endogenous link strength poses new conceptual difficulties, which were absent in the literature where links have a fixed, exogenous value. First, the technology transforming individual investments into the quality of a bilateral link needs to be specified. In this exploratory analysis of the formation of links with endogenous quality, we

\footnotetext{
${ }^{1}$ See Goyal (2005), which also emphasizes the importance of studying networks where the strength of links can be chosen endogenously.

${ }^{2}$ The role of social networks as communication devices has long been acknowledged. The use of social networks in job referrals has been studied, among others, by Granovetter (1974), Boorman (1975), Montgomery (1991), Calvo-Armengol and Jackson (2004). A classic reference on the role of social networks in the diffusion of innovation is Coleman, Katz and Menzel (1966).
} 
focus attention on the simple situation where agents' investment decisions are independent. We also assume that agents may face a fixed cost in the formation of links. This leads us to specify the strength of a link as an additively separable and convex function of agents' investments. In this setting, agents do not need the consent of their partner to form a link, and the model can be interpreted as a generalization of Bala and Goyal (2000)'s version of one-sided, two-way flow model of link formation. In a later Section of the paper, we also provide a partial analysis of the case of perfect complements, where the strength of a link is given by the minimum of both parties' investments. This case is reminiscent of Jackson and Wolinsky (1996)'s model of link formation with consent, and our analysis generalizes their approach to handle weighted networks where agents optimally "match" investments on their bilateral link.

The second difficulty stems from the modeling of benefits from indirect connections. In the existing literature, it is assumed that agents always choose to connect through the shortest path, and that indirect benefits are a decreasing function of the length of the connection. ${ }^{3}$ When link strength is endogenous, it is natural to suppose that agents choose to connect through the path with the highest reliability (measured by the product of link strengths normalized to belong to $(0,1))$ - which is not necessarily the shortest path. However, this is not the only way to model the reliability of an indirect connection. At the end of the paper, we consider an alternative model, where the value of an indirect connection depends on the weakest link along the path.

Throughout the paper, we consider the following problem. We suppose that agents are endowed with a fixed endowment $X$, that they allocate across different connections. Our first task is to compute the strongly efficient network architecture, which maximizes the sum of benefits of all agents. In a second step, we characterize the set of stable networks, obtained when agents form relations in a voluntary, decentralized manner. Because of the well-known coordination problem in noncooperative games of bilateral link formation, we consider two equilibrium concepts. We say that a network is Nash stable if it is immune to individual deviations, and strongly pairwise stable if it is immune to deviations by individuals as well as pairs of agents.

\footnotetext{
${ }^{3}$ Jackson and Wolinsky (1996) specify a decay factor $\delta \in(0,1)$ and assume that indirect benefits are given by $\delta^{t} v$ where $t$ is the length of the shortest path connecting two agents and $v$ a fixed parameter. For most of their analysis, Bala and Goyal (2000) suppose that the value of a connection is independent of the distance between two agents.
} 
The main results of our study can be summarized as follows. We first show that the efficient network must be a star, that is a network where one agent (the hub) is connected to all other agents, while peripheral agents are only connected with the hub. Moreover, when link strength is a linear function of individual investments, the unique efficient network is the symmetric star in which the hub invests equally on all the links.

We also have a characterization of stable networks. If link strength is a strictly convex function of individual investments, then the unique Nash stable network is the star where the center of the star invests fully on just one link. If link strength is a linear function of individual investments, then only stars can be strongly pairwise stable. We provide a necessary and sufficient condition for the existence of strongly pairwise stable networks in terms of $X$ and the number of individuals. The larger the number of individuals, the weaker is the sufficient condition.

In the penultimate section of the paper, we consider two extensions of the basic framework. First, we retain the assumption that link strength is a separable, convex function of individual investments, but consider a model of weakest-link reliability, where the value of an indirect connection is independent of the distance, and is only affected by the strength of the weakest link along the path. In this case, the symmetric star again emerges as an efficient network architecture. ${ }^{4}$ The symmetric star is also strongly pairwise stable, as any deviation from a network where all links have equal strength is bound to decrease the value of the weakest link.

Second, we consider the case where individual investments act as perfect complements in the "production" of link strength. The efficient network architecture is now very different. Trees cannot be optimal, because end players of the network waste part of their endowments, and have an incentive to invest what remains from their endowment on a new link. Regular graphs (where all agents have the same number of links) are likely candidates for efficient network architectures, and we show that for small numbers of players, the circle is in fact the efficient network architecture. However, when the number of players increases, the circle can be dominated by other network architectures. Finally, we note that the circle is always strongly pairwise stable.

Our results are related to the results obtained in the discrete link for-

\footnotetext{
${ }^{4}$ However, this is not the unique efficient architectures - other networks which generate the same distribution of link strengths are equally efficient.
} 
mation literature (Bala and Goyal (2000)'s two-way flow model with decay, Hojman and Szeidl (2007)'s model with strong decreasing returns to scale and decay and Feri (2007)'s evolutionary model) and we now comment on the relation between our analyses. Bala and Goyal (2000) also characterize the star as the efficient network architecture in their two-way flow model with decay when the cost parameter is such that neither the empty nor the complete graph are efficient (Bala and Goyal (2000), Proposition 5.5 p. 1220). However, Nash equilibrium has little predictive power in their model, and even when they resort to the refinement of strict Nash equilibria, they are unable to obtain a complete characterization in the two-way flow model with decay (see Bala and Goyal (2000), Proposition 5.3 p. 1215).) By placing additional restrictions on the benefit function and the effect of decay, Hojman and Szeidl (2007) are able to fully characterize the set of Nash equilibria, and show that it is a periphery-sponsored star (Hojman and Szeidl (2007), Theorem 1.) However, the characterization of efficient networks in their model is complicated (see the Example 1 and Proposition 1 in Hojman and Szeidl (2007)). Feri (2007) also characterizes periphery-sponsored stars as the unique stochastically stable equilibria of his model.

The similarity between these characterizations and our results are partly driven by our assumption on the link formation technology. Because the formation of links typically involves a fixed cost, we find natural to assume that link strength is a convex function of individual investments. As a consequence, agents have an incentive to concentrate their investments on a single link, and the model appears to be similar to a model of discrete link formation, where every agent forms a single link of maximal intensity. However, there remain significant differences between our model of endogenous link quality and discrete link formation models. First, because we consider a richer set of weighted networks, agents have more opportunities. To establish that the star is the efficient network in our setting is a much harder task than in Bala and Goyal (2000)'s analysis, because we are optimizing over a much larger set of feasible networks. Similarly, in the noncooperative game of link formation, we characterize best responses in a larger space of strategies. Second, in our setting, the fixed cost can be made arbitrarily low, and we devote much attention to the case of linear investments where the fixed cost is equal to zero. With linear investments, agents have no a priori reason to concentrate their investments on a single link, and the emergence of equilibrium networks where some agents invest all their resources in a single link is not driven by the assumption on technology but by the general structure of the 
model. Finally, in our analysis, the concentration of investments in a single link is an equilibrium result rather than an assumption. As we will argue below, the fact that agents could have invested in multiple links changes the analysis deeply, and is the driving force behind our sharp characterization of Nash and strongly pairwise equilibria of the game of link formation.

\section{Related Work}

Given the obvious importance of networks with links of varying strength, a number of recent papers have proposed models where agents choose how much to invest in a relationship. In some of these models, agents choose their investment after the network has been established. For example, Bramoulle and Kranton (2006) study the agent's incentives to provide a public good once the network is fixed. In a specific model of strategic alliances among firms, Goyal and Moraga-Gonzales (2001) consider a two-stage model where firms first form links and then decide their R\&D investment. This is a model of "nonspecific networking" because the firm chooses the same investment across all its links. Durieu, Haller and Solal (2004) also consider a model of nonspecific networking. Agents choose a single investment, which applies to the links with all other agents. Still in the framework of nonspecific networking, Cabrales, Calvo-Armengol and Zenou (2007) propose an axiomatic derivation of the relation between pairwise link intensities and agents' "socialization intensities", represented by scalars. Brueckner (2003) considers a model of friendship networks. Agents choose to invest in relationships, and the value of indirect benefits is given by the product of the strength of links. For most of his analysis, Brueckner (2003) concentrates on three player networks, and studies the effect of the network structure on the investment choices in the complete and star networks. Other papers, more closely related to ours, consider the formation of the network and the choice of investments as simultaneous. Goyal, Konovalov and Moraga-Gonzales (2003) extend their analysis of cost-reducing alliances by allowing firms to choose different investments on different links. Rogers (2005) proposes a different model of network formation with endogenous link quality. In his model, links are directed and can be interpreted as the influence that every agent has on another agent. As in our paper, agents allocate a fixed endowment on different relationships. Agent's utilities depend on the values of other agents in their neighborhoods, and are defined in a circular way. Two different models are studied: one where agents receive value from their neighbors, and one where they give values to their neighbors. In this environment, which is very 
different from ours, Rogers (2005) characterizes Nash and efficient network structures, emphasizing the importance of heterogeneity across agents.

\section{Model and Notations}

\section{Investments and link strength}

Let $N=\{1,2, \ldots, n\}$ be a set of individuals. Individuals derive benefits from links to other individuals. These benefits may be the pleasure from friendship, or the utility from (non-rival) information possessed by other individuals, and so on. In order to fix ideas, we will henceforth interpret benefits as coming from information possessed by other individuals. Each individual has a total resource (time, money) of $X>0$, and has to decide on how to allocate $X$ in establishing links with others. ${ }^{5}$

Let $x_{i}^{j}$ denote the amount of resource invested by player $i$ in the relationship with $j$. Then, the strength of the relationship between $i$ and $j, s_{i j}$ is assumed to be a symmetric, additively separable function of $x_{i}^{j}$ and $x_{j}^{i}$,

$$
s_{i j}=\phi\left(x_{i}^{j}\right)+\phi\left(x_{j}^{i}\right)
$$

where $\phi($.$) is a nondecreasing, convex function. Furthermore, we suppose$ that $\phi(0)=0$ and $\phi(X)<1 / 2$ so that $s_{i j} \in(0,1)$.

Some remarks are in order. First, we consider a setting where link strength is an additively separable function of investments. This implies that an agent's decision to allocate his endowment over direct links is independent of his neighbors' decisions. However, this does not mean that an agent's investment strategy is independent of the choices of other agents, as these choices affect the value of indirect links and hence the payoffs obtained in the game. Second, we consider a model with nondecreasing returns to investment. While this assumption may seem at odds with the classical literature on productive investments, we strongly believe that convexity is the right assumption to make when one discusses investments in communication links. The formation of any network involves a fixed cost component. Whenever agents need to invest a fixed initial amount in a communication link, the quality of the communication link is likely to be a convex function of investments. In fact, the literature on discrete link formation assumes an extreme form of

\footnotetext{
${ }^{5}$ To keep matters simple, we thus assume that agents do not incur a monetary cost of investment, but only an opportunity cost.
} 
convexity, where $\phi\left(x_{i}^{j}\right)=0$ as long as $x_{i}^{j}<c$ and $\phi\left(x_{i}^{j}\right)=\bar{s}$ when $x_{i}^{j} \geq c$. Finally, without loss of generality, we can normalize units of investment, so that every link strength is a number between 0 and 1 .

\section{Link strength and reliability}

We say that individuals $i$ and $j$ are linked if and only if $s_{i j}>0$. Each pattern of allocations of $X$, that is the vector $\mathbf{x} \equiv\left(x_{i}^{j}\right)_{\{i, j \in N, i \neq j\}}$ results in a weighted graph, which we denote by $g(\mathbf{x}) .{ }^{6}$ We say that $i j \in g(\mathbf{x})$ if $\min \left(x_{i}^{j}, x_{j}^{i}\right)>0$.

Given any $g$, a path between individuals $i$ and $j$ is a sequence $i^{0}=$ $i, i^{1}, \ldots, i^{m}, \ldots, i^{M}=j$ such that $i^{m+1} i^{m} \in g$ for all $m \in\{0, \ldots, M-1\}$. Two individuals are connected if there exists a path between them. Connectedness defines an equivalence relation, and we can partition the set of individuals according to this relation. Blocks of that partition are called components.

Suppose $i$ and $j$ are connected. Then, the benefit that $i$ derives from $j$ depends on the reliability with which $i$ can access $j$ 's information. There are different ways to model how the strength of links affects the reliability of the communication channel. In our view, the most natural interpretation is that the strength of a link is an index of the quality of the transmission, so that messages sent along stronger links are more likely to be delivered without delay or distortion. With this interpretation, the reliability of any path between $i$ and $j$ is given by the product of the link strengths along the path. For any path $p(i, j)=i, i^{1}, \ldots, i^{M-1}, j$, we thus define

$$
r(p(i, j))=s_{i i^{1} \ldots} \ldots s_{i^{m-1}} i^{m} \ldots s_{i^{M-1} j} .
$$

We assume that agents always choose to transmit information along the path with the highest reliability, and for any connected pair of agents $i, j$, we let $p^{*}(i, j)$ denote the most reliable path in the set of all paths $P(i, j)$

$$
p^{*}(i, j)=\arg \max _{p(i, j) \in P(i, j)} r(p(i, j)) .
$$

The benefit of the connection from $i$ to $j$ is then given by

$$
R(i, j)=r\left(p^{*}(i, j)\right)=\max _{p(i, j) \in P(i, j)} r(p(i, j))
$$

\footnotetext{
${ }^{6}$ To simplify notation, we will sometimes ignore the dependence of $g$ on the specific pattern of allocations.
} 
The total utility that agent $i$ obtains in the weighted graph $g$ can then be computed as:

$$
U_{i}(g)=\sum_{j \neq i} R(i, j)
$$

and the total value of the graph is given by

$$
V(g)=\sum_{i} U_{i}(g)
$$

\section{Efficient and stable networks}

We now define efficient and stable graphs. The notion of efficiency that we use is the strong efficiency notion introduced by Jackson and Wolinsky (1996).

Definition 1 A graph $g$ is efficient if $V(g) \geq V\left(g^{\prime}\right)$ for all $g^{\prime}$.

We now describe the concepts of stability that will be used in this paper.

Given any pattern of investments $\mathbf{x}$, and individual $i,\left(\mathbf{x}_{-\mathbf{i}}, x_{i}^{\prime}\right)$ denotes the vector where $i$ deviates from $x_{i}$ to $x_{i}^{\prime}$. Similarly, $\left(\mathbf{x}_{-\mathbf{i}, \mathbf{j}}, x_{i, j}^{\prime}\right)$ denotes the vector where $i$ and $j$ have jointly deviated from $\left(x_{i}, x_{j}\right)$ to $\left(x_{i}^{\prime}, x_{j}^{\prime}\right)$.

Definition $2 A$ graph $g(\mathbf{x})$ is Nash stable if there is no individual $i$ and $x_{i}^{\prime}$ such that $U_{i}\left(g\left(\mathbf{x}_{-\mathbf{i}}, x_{i}^{\prime}\right)\right)>U_{i}(g(\mathbf{x}))$.

So, a graph $g$ induced by a vector $\mathbf{x}$ is Nash stable if no individual can change her pattern of investment in the different links and obtain a higher utility.

Definition 3 A graph $g(\mathbf{x})$ is strongly pairwise stable if it is Nash stable and there is no pair of individuals $(i, j)$ and joint deviation $\left(x_{i}^{\prime}, x_{j}^{\prime}\right)$ such that

$$
U_{k}\left(g\left(\mathbf{x}_{-\mathbf{i}, \mathbf{j}}, x_{i, j}^{\prime}\right)\right)>U_{k}(g(\mathbf{x})) \text { for } k=i, j
$$

A Nash-stable graph is strongly pairwise stable if no pair of individuals can both be strictly better off by changing their pattern of investment. Jackson and Wolinsky (1996) define a weaker notion of stability - pairwise stability. They basically restrict deviations by assuming that only one link at 
a time can be changed. Our current definition corresponds to the definition of pairwise stability used by Dutta and Mutuswami (1997). ${ }^{7}$

We also recall that a tree is an acyclic connected network, (a connected network for which there does not exist a sequence of nodes $i_{0}, i_{1}, \ldots, i_{n}$ such that $i_{k} i_{k+1} \in g$ for $i=0, n-1$ and $i_{0}=i_{n}$ ). Among trees, a particular network structure is the star.

Definition 4 A graph $g$ is a star if there is some $i \in N$ such that $g=$ $\{i k \mid k \in N, k \neq i\}$.

The distinguished individual $i$ figuring in the definition will be referred to as the "hub".

\section{Efficient and stable networks}

In this Section, we characterize the set of efficient and stable networks, and provide some intuition for our results. The formal proofs are given in the Appendix. Our first Theorem characterizes efficient networks.

Theorem 1 Suppose $\phi$ is a separable, convex function of individuals investments. Then, the unique efficient network is a star. Moreover, if $\phi$ is linear, then the unique efficient network is the symmetric star where the hub invests an equal amount in all links with peripheral agents.

The intuitive explanation for this result is the following. The star is a minimally connected network, every peripheral agent concentrates his investment on a single link, and the distance between two nodes which are not directly connected is minimized. All these features contribute to making the star an obvious candidate for the efficient network. In fact, Bala and Goyal (2000) also show that the star is the unique efficient network architecture in the discrete link formation model. The novelty of Theorem 1 is that the set of networks on which we optimize is much richer than in Bala and Goyal (2000), as we allow agents to use weighted links. Hence, the main message

\footnotetext{
${ }^{7}$ The concept of "strong pairwise stability" has been used by different authors under different names. See Gilles and Sarangi (2004) and Bloch and Jackson (2006) for an attempt to unify the terminology and a comparison of different stability concepts.
} 
of the Theorem is that stars remain the unique efficient networks even if we consider a much larger set of weighted networks. Because the set of networks on which we optimize is much larger, the proof of Theorem 1 is markedly different, and much more involved than the proof of Theorem 5.5 in Bala and Goyal (2000).

In the proof, we first show that, by reducing the number of links to form a star, the aggregate benefits of the network increase. By convexity, links become stronger, and the distance between nodes is reduced. However, the star that is formed is not necessarily feasible - it could involve the hub investing more than her endowment $X$. To solve this problem, we gradually reallocate the investments on the links in a way which increases the value of aggregate benefits. Finally, we show that the value of the network increases when we merge two stars into a single one.

Our second Theorem shows that the star is also the unique Nash stable network for strictly convex investments, and the only candidate for strongly pairwise stable networks with linear investments.

Theorem 2 (i) If $\phi$ is strictly convex, the unique Nash stable network is a star where the hub invests all her endowment in a single link.

(ii) If $\phi$ is linear, a strongly pairwise stable network must be a star with $(n-1)$ peripheral nodes, and the set of strongly pairwise stable networks is nonempty iff $X \geq \frac{(n-1)^{2}}{n\left(n^{2}-3 n+3\right)}$.

Theorem 2 characterizes the set of Nash stable networks when $\phi$ is strictly convex, and strongly pairwise stable networks for linear $\phi$. In both cases, stable networks are stars. With strictly increasing returns to scale, the hub invests in a single link to a peripheral agent; for constant returns to scale, the allocation of investment of the hub is indeterminate. The efficient symmetric star is stable as long as the endowment $X$ is greater than $\frac{(n-1)^{2}}{n\left(n^{2}-3 n+3\right)}$, an expression which is decreasing in $n$ for $n \geq 3$, and converges to 0 as $n$ goes to infinity.

In contrast to Bala and Goyal (2000), we characterize the star as the unique Nash stable architecture with a convex technology, and the unique candidate for strongly pairwise equilibrium with a linear technology. The strategy of the proof is related to Hojman and Szeidl (2007)'s proof even though the models and the arguments are different. With a strictly convex 
link strength technology, we show that agents never have an incentive to invest in multiple links. Given this "one-link property", we progressively rule out all network architectures but the star. ${ }^{8}$

We first rule out cycles in equilibrium. Cycles arise when all agents invest fully on the link to their neighbor. Agents must then access the same indirect benefits through any node in the cycle, so that the cycle is fully symmetric. An agent then has an incentive to redirect his investment towards the neighbor who invests towards him, breaking the cycle towards a line but increasing the strength of his direct link.

Once cycles are ruled out, we show that the only candidate equilibrium among trees are stars. For any tree with diameter greater than or equal to three, we prove that there must exist terminal nodes who have an incentive to reallocate their investment in order to decrease the distance of their indirect connections. Finally, we provide an argument to show that disconnected stars cannot form in equilibrium.

When the link strength technology is linear, agents may invest in multiple links, and the marginal benefits of any connection must be equalized. We use this fact to construct joint pairwise deviations, and are able to show that, in any strongly pairwise equilibrium, if an agent invests in multiple links, all his neighbors must reciprocate by investing their entire endowment towards him. ${ }^{9}$ This characterization enables us to use the same steps as in the case of strictly convex link strength technologies to rule out all network architectures but stars.

Taken together, theorems 1 and 2 also help us understand the gap between efficiency and stability in networks with endogenous link strength. Efficient and stable networks are always stars, but the allocation of the hub's investment may differ. With increasing returns to investment, the hub invests all his investment in one link in equilibrium, but efficiency may require him to spread his investment across links, in order to increase the benefits of peripheral agents. When $\phi$ is linear, the symmetric star emerges as a strongly pairwise stable network when endowments are large enough. Since this is the unique efficient network, this result identifies a condition under

\footnotetext{
${ }^{8}$ This is also the structure of Hojman and Szeidl (2007)'s proof, which first establishes that agents invest in a single link (Lemma 2), then shows that the distance between terminal nodes is at most two (Lemma 3) and finally rules out cycles and paths (Lemma 4).

${ }^{9}$ Notice that this argument exploits the fact that individual investment choices are continuous, and has no equivalent in discrete models of link formation.
} 
which efficiency is compatible with stability when individual investments are perfect substitutes. It is interesting to note that the threshold value of $X$ required to ensure this compatibility becomes smaller and smaller when the number of individuals becomes large.

\section{Extensions}

In this section, we discuss two extensions of the analysis, one dealing with an alternative model of investment, and the other with an alternative model of reliability.

\subsection{Weakest link reliability}

We now suppose that agents evaluate paths according to the value of the weakest link in the path rather than the product of link strengths. This notion of "weakest link reliability" is useful for physical communication networks, like the internet, where the quality of a connection depends on the bottleneck of the network. Formally, we define the alternative notion of reliability as:

$$
\widehat{r}(p(i, j))=\min _{s_{i^{m-1} i^{m} \in p(i, j)}} s_{i^{m-1} i^{m}}
$$

Agents choose to use the paths with the highest reliability, and we define the benefit of a connection from $i$ to $j$ in this setting as:

$$
\widehat{R}(i, j)=\max _{p(i, j) \in P(i, j)} \min _{s_{i^{m-1} i^{m}} \in p(i, j)} s_{i^{m-1} i^{m}}
$$

With weakest link reliability, distance between nodes becomes irrelevant. Networks with different architectures but with identical distributions of link strengths result in the same value. Hence, efficient networks will typically not be unique. The next Theorem shows that any efficient network with weakest link reliability is equivalent to a star - the unique efficient network in our baseline model.

Theorem 3 Let $\phi$ be a separable, convex function of individual investments, and suppose indirect benefits correspond to the notion of weakest link reliability. Then, 
(i) Any efficient network is equivalent to a star.

(ii) If $\phi$ is linear, then any efficient network is equivalent to a symmetric star where the hub invests equal amounts on every link. Moreover, the symmetric star is strongly pairwise stable.

(iii) If $\phi$ is strictly convex, a star where the hub invests in a single link is strongly pairwise stable.

The characterization of stars as efficient networks with weakest link reliability relies on arguments which are very similar to those showing that stars are optimal in our baseline model. The main difference between the two approaches is that efficient network architectures with weakest link reliability are not unique. In fact, when link strength technology is linear, any tree with links of equal strength is efficient, and it can be shown that links of equal strength can be obtained for any tree. ${ }^{10}$ As stars are strongly pairwise stable, the set of strongly pairwise stable networks is nonempty.

A complete characterization of the set of stable networks is difficult. Since the value of a path depends on the weakest link along the path, a joint reallocation of investments by two players leading to more equal link strengths does not necessarily increase their payoffs. This lack of responsiveness of payoffs to individual choices prevents deviations and results in a large number of strongly pairwise stable networks. The following example illustrates why only a global reallocation of investments can improve individual payoffs.

Example 1 Let $\# N=6$. Consider a line where $s_{i, i+1}>0$ and $s_{i j}=0$ if $j \neq i+1$. In particular, let $s_{12}=s_{56}=5 / 4 X$, and $s_{23}=s_{34}=s_{45}=7 / 6 X$.

All individuals can gain if 2,3,4 and 5 relocate their investments so as to equalize link strength to $6 / 5 X$. However, this joint deviation requires coordination among more than two agents and is not possible given our equilibrium concept. No pair of agent has an incentive to deviate from their strategies, so this inefficient network is strongly pairwise stable.

\footnotetext{
${ }^{10}$ To prove this last statement, one has to construct an algorithm, where terminal nodes invest their full endowment on their predecessor, who invest $X /(n-1)$ on their successors and $(n-2) X /(n-1)$ on their predecessors, etc..
} 


\subsection{Investments as perfect complements}

As a polar opposite to the case of perfect substitutes, we consider a model where investments are perfect complements, so that an agent only benefits from a relationship when the other agent also invests in the link. Formally,

$$
s_{i j}=\min \left\{x_{i}^{j}, x_{j}^{i}\right\} .
$$

When investments are perfect complements, agents should allocate "matching" investments on every link in order to maximize direct benefits. This intuition suggests that the efficient and stable network architectures will be very different from those obtained in the previous section. Stars will perform very badly, because the hub can only invest a small amount on every link. In contrast, regular networks where all agents invest the same amount on every link should perform fairly well.

Unfortunately, once indirect benefits are taken into account, the analysis of efficient and stable networks becomes intractable. We have to content ourselves with partial characterization results summarized in the following Theorem.

Theorem 4 Suppose that individual investments are perfect complements. Then,

(i) An efficient graph cannot contain any component with three or more nodes which is a tree.

(ii) For $3 \leq n \leq 7$, the symmetric circle where every link has value $X / 2$ is the unique efficient network.

(iii) Moreover, the symmetric circle is strongly pairwise stable.

Theorem 4 establishes that trees cannot be efficient, and for small numbers of players (where distance does not matter too much), the unique regular network of degree 2 is the efficient network. However, for larger numbers of agents, efficiency may require a denser graph, where links are weaker, but distances between nodes shorter. Finally, we check that the set of strongly pairwise networks is nonempty and contains the symmetric circle. ${ }^{11}$ Hence,

\footnotetext{
${ }^{11} \mathrm{~A}$ complete characterization of the strongly pairwise stable networks is a difficult task. Since investments on a link are strategic complements, multiple equilibria typically arise, making the analysis of the entire game intractable.
} 
comparing the case of perfect substitutes and perfect complements, we see that in the latter case, efficient and stable networks will be denser and more symmetric across players.

\section{Conclusion}

In this paper, we analyze the formation of communication networks when players choose how much to invest in each relationship. We suppose that players have a fixed endowment that they can allocate across links, and in the baseline model, suppose that link strength is an additively separable and convex function of individual investments, and that agents use the path which maximizes the product of link strengths. Under these assumptions, we characterize the optimal and stable networks. We also provide partial characterization results for alternative specifications of the investment technology and the benefit function.

In our view, this paper provides a first step in the study of networks where agents endogenously choose the quality of the links they form. One obvious drawback of our analysis is that agents are ex ante homogeneous. This assumption leads us to conclude that links will all be of the same quality (in the case of linear investments), or that, due to the hub's investments, some agents will be better connected than others (in the case of convex investments). Neither of these distributions of link intensities does justice to the broad array of social networks one observes in reality. In order to study the formation of networks with varying link quality, and the effect of individual characteristics on the efficient and stable distribution of link qualities, we need to introduce heterogeneity across agents. Following Rogers (2005), we could consider agents who differ both in their attractiveness (the intrinsic utility they bring to other agents), and their endowment. This seems to us to be a very promising avenue for future work.

\section{References}

Bala, V. And S. Goyal (2000) "A Noncooperative Model of Network Formation," Econometrica 68, 1181-1229.

Bloch, F. And M. O. JaCKson (2006) "Equilibrium Definitions in Network Formation Games", International Journal of Game Theory 34, 305-318. 
Boorman, S. (1975) "A Combinatorial Optimization Model for Transmission of Job Information through Contact Networks", Bell Journal of Economics 6, 216-249.

Bramoulle, Y. And R. Kranton (2007) "Public Goods in Networks", Journal of Economic Theory 135, 478-494.

BRUECKNER, J. (2003) "Friendship Networks", mimeo., University of Illinois at Urbana Champaign.

Cabrales, A., Calvo-Armengol, A. And Y. Zenou (2007) "Effort and Synergies in Network Formation", mimeo., Universidad Carlos III, Universitat Autonoma de Barcelona, IUI Stockholm.

Calvo-Armengol, A. And M. Jackson (2004) "The Effects of Social Networks on Employment and Inequality" American Economic Review 94, 426-454.

Coleman, J., E. Katz And H. Menzel (1966) Medical Innovation: A Diffusion Study, Bobbs Hill, New York.

Durieu, J., H. Haller And P. Solal (2004) "Nonspecific Networking", mimeo., Virginia Tech.and University of Saint-Etienne.

Dutta, B. And S. Mutuswami (1997) "Stable Networks", Journal of Economic Theory 76, 322-344.

FerI, F. (2007) "Stochastic Stability in Networks with Decay", Journal of Economic Theory 135, 442-457.

Gilles, R. And S. Sarangi (2004) "Social Network Formation with Consent", mimeo., Virginia Tech and University of Louisiana.

Goyal, S. (2005) "Strong and Weak Links", Journal of the European Economic Association, 3, 608-616. .

Goyal, S. And J. Moraga Gonzales (2001) "R\&D Networks", RAND Journal of Economics 32, 686-707.

Goyal, S., A. Konovalov and J. Moraga Gonzales (2003) "Hybrid R\&D Networks,", mimeo., Tinbergen Institute.

Granovetter, M. (1974) Getting a job: A Study of Contacts and Careers, Harvard University Press, Cambridge.

Hojman, D. And A. Szeidl (2007) "Core and Periphery in Networks", Journal of Economic Theory, forthcoming.

JaCkson, M. O. And A. Wolinsky (1996) "A Strategic Model of Economic and Social Networks," Journal of Economic Theory 71, 44-74.

Montgomery, I. (1991) "Social Networks and Labor Market Outcomes" (1991) American Economic Review 81, 1408-1418. 
Rogers, B. (2005) "A Strategic Theory of Network Status," mimeo., MEDS, Northwestern University.

\section{Appendix}

Proof of Theorem 1: We prove the first statement in two steps. Consider any feasible component $h$ of $g$ of size $m$ where the total amount of investment is $m X{ }^{12}$

Step 1: We construct a feasible star $S$ with higher aggregate utility than $h$, whenever $h$ is not a star.

Step 2: If the graph $g$ contains different components, we construct a single connected star which has higher aggregate utility than the sum of the stars.

Proof of Step 1: Order the link strengths of the component $h$ so that:

$$
z_{1} \geq z_{2} \geq \ldots \geq z_{K}
$$

Construct a star $S$ by picking an agent at random (say agent $m$ ), and connecting him to the $(m-1)$ other agents with links of strengths $z_{1}, z_{2}, \ldots, \sum_{k=m-1}^{K} z_{k}$ in the following way:

$$
\begin{aligned}
& \text { for all } i=1, \ldots, m-2, x_{i}^{m}=\min \left\{\phi^{-1}\left(z_{i}\right), X\right\}, x_{m}^{i}=\phi^{-1}\left(z_{i}\right)-x_{i}^{m} \\
& x_{m-1}^{m}=\min \left\{\phi^{-1}\left(\sum_{k=m-1}^{K} z_{k}\right), X\right\}, x_{m}^{i}=\phi^{-1}\left(\sum_{k=m-1}^{K} z_{k}\right)-x_{m-1}^{m}
\end{aligned}
$$

Let $s_{i}, i=1, \ldots, m-1$, denote the strengths of the $(m-1)$ links in the star. Notice that by construction,

$$
s_{i}=z_{i}, i=1, \ldots, m-2, \text { and } s_{m-1} \geq z_{m-1}
$$

In this star, direct benefits are exactly equal to those of the component $h$. We show that indirect benefits have increased. In the star $S$, indirect benefits are given by:

$$
I=2 \sum_{i \neq j, i, j=1}^{m-1} s_{i} s_{j}
$$

\footnotetext{
${ }^{12}$ If the total amount invested in the component is strictly smaller than $m X$, then clearly the sum of utilities of agents in the component cannot be maximal.
} 
Let $D=\{i j \mid i$ and $j$ are not neighbours in $h\}$. So, $D$ is the set of pairs of nodes which are not directly connected in $h$, and so derive indirect benefits from each other. ${ }^{13}$

Suppose first that $h$ is a tree, but not a star. For each pair $i, j$ in $D$, let $z_{t_{i}}$ and $z_{t_{j}}$ denote the strengths of the two terminal links in the unique path $p^{*}(i, j)$. Clearly, for all $i, j \in D$,

$$
R^{p}(i, j) \leq z_{t_{i}} z_{t_{j}}
$$

Moreover, since $h$ is not a star, the (geodesic) distance between at least one pair of nodes, say $i, j$ in $h$ must be at least three. Hence, the inequality must be strict for such $i, j$ since the maximum strength of any link is strictly less than one and the indirect benefit is the product of link strengths along the most reliable path. Also, note that each pair of nodes in $D$ is associated with a unique pair of terminal links, and that one can construct exactly $\frac{(m-1)(m-2)}{2}$ pairs of terminal nodes out of the set $\left\{z_{1}, \ldots, z_{m-1}\right\}$. So, letting $I^{\prime}$ denote the sum of indirect benefits in $h$, the following inequality must be true

$$
I^{\prime}<2 \sum_{i \neq j, i, j=1}^{m-1} z_{i} z_{j}=I
$$

where the last equality follows from equation 1.

Suppose now that $h$ is not a tree, so that the cardinality of $D$ is now strictly less than $\frac{(m-1)(m-2)}{2}$. We can again associate a unique pair of terminal links $\left(z_{t_{i}}, z_{t_{j}}\right)$ to any pair of nodes $i, j \in D .{ }^{14}$ Equation 2 will hold again, aggregate indirect benefits in $h$ is $I^{\prime}$, where

$$
I^{\prime}<2 \sum_{i \neq j, i, j=1}^{m-1} z_{i} z_{j} \leq I
$$

The first inequality holds because there are now fewer than $\frac{(m-1)(m-2)}{2}$ pairs in $D$, and the sum is being taken over the product of the pairs which can be formed out of the strongest $(m-1)$ links.

If the star $S$ is feasible, then this completes the proof. However, $S$ may not be feasible. By construction, each peripheral agent invests no more than $X$. However,

\footnotetext{
${ }^{13}$ Note that if $i j \in h$, but the link strength is so weak that they do not derive direct benefits from each other, then $h$ cannot be efficient; both $i$ and $j$ should switch their investment from $i j$ to some other link.

${ }^{14}$ Since $h$ is not a tree, there may be more than one most reliable path connecting $i$ and $j$. The tie-breaking rule used to select some most reliable path - and hence the terminal links - is not important since the pair $\left(z_{t_{i}}, z_{t_{j}}\right)$ can only be terminal links for the pair $(i, j)$.
} 
the construction may involve the center $m$ investing more than $X .{ }^{15}$

We now show how to construct a feasible star

from $S$ which will have at least as large direct and indirect benefits as $S$, and hence strictly larger aggregate benefits than $h$.

Claim 1 Consider the star $S$ constructed above. If, for all $i=1, \ldots, m-1$, $x_{i}^{m}=X$, then $\sum_{i=1}^{m-1} x_{m}^{i} \leq X$, and so the star $S$ is feasible.

Proof of Claim 1: Suppose all peripheral agents invest $X$ on the link with $m$. Then,

$$
\begin{aligned}
\sum_{i=1}^{m-1} x_{m}^{i} & =\sum_{i=1}^{m-2} \phi^{-1}\left(z_{i}\right)+\phi^{-1}\left(\sum_{k=m-1}^{K} z_{k}\right)-(m-1) X \\
& \leq \sum_{i=1}^{K} \phi^{-1}\left(z_{i}\right)-(m-1) X \\
& \leq X
\end{aligned}
$$

We thus observe that, if star $S$ is not feasible, there must exist a link $k$ such that $x_{k}^{m}<X$. Of course, $x_{m}^{k}=0$.

Choose any $i$ such that $x_{m}^{i}>0$. Consider the new star $\bar{S}$ where the only change from $S$ is that $\bar{x}_{k}^{m}=\min \left\{X, x_{k}^{m}+x_{m}^{i}\right\}$, and $\bar{x}_{m}^{i}=x_{m}^{i}-\left(\bar{x}_{k}^{m}-x_{k}^{m}\right)$. Let $\bar{s}_{k}-s_{k}=\epsilon$ and $s_{i}-\bar{s}_{i}=\delta$. Then, $\epsilon \geq \delta$ from convexity of $\phi$. That is, this transfer of resource from link $i$ to link $k$ must (weakly) increase aggregate direct benefit.

We now check the effect of this change on indirect benefits. Let $\bar{I}$ and $I$ be the aggregate indirect benefit in $\bar{S}$ and $S$ respectively. Then,

$$
\begin{aligned}
\bar{I}-I & =2\left[(\epsilon-\delta) \sum_{j \neq i, k} s_{j}+\left(s_{k}+\epsilon\right)\left(s_{i}-\delta\right)-s_{k} s_{i}\right] \\
& \geq 2\left(\epsilon s_{i}-\delta s_{k}-\epsilon \delta\right) \\
& \geq 2 \epsilon\left(s_{i}-s_{k}-\delta\right) \\
& =2 \epsilon\left(\bar{s}_{i}-s_{k}\right) \\
& \geq 0
\end{aligned}
$$

\footnotetext{
${ }^{15}$ However, the feasibility of $h$ and convexity of $\phi$ ensures that the total resource used up in $S$ does not exceed $m X$.
} 
The last inequality holds because $\bar{s}_{i}=\phi(X)+\phi\left(\bar{x}_{m}^{i}\right) \geq \phi(X) \geq s_{k}$.

So, aggregate benefit is at least as high in $\bar{S}$ as it is in $S$. If $\bar{S}$ is not feasible, then we can continue to transfer resources from the hub to some peripheral node in the same way, until some star with aggregate benefit at least as high as $S$ is feasible.

Proof of Step 2: Consider two feasible stars $S_{1}$ and $S_{2}$ of sizes $s_{1}$ and $s_{2}$. Construct a new star $S^{*}$ of size $s_{1}+s_{2}$ centered around the hub of $S, m_{2}$, with the following investments:

$$
\begin{aligned}
x_{i}^{m_{2 *}} & =X \text { for all } i \neq m_{2} \\
x_{m_{2}}^{i *} & =x_{m_{2}}^{i}
\end{aligned}
$$

In terms of direct benefits, the only change between the new star $S^{*}$ and the stars $S_{1}$ and $S_{2}$ is that the hub of $S_{1}$ now invests fully on its link with $m_{2}$. Given the convexity of $\phi$, this change must have weakly increased aggregate direct benefits. Consider next the indirect benefits in the new star, $I^{*}$ and the sum of indirect benefits in the two stars, $I_{1}+I_{2}$. Indirect benefits inside the star $S_{2}$ have not changed, and peripheral nodes of $S_{2}$ have gained access to new indirect connections. Agents in the star $S_{1}$ have gained access to new indirect connections to agents in star $S_{2}$. We focus attention on the difference in aggregate indirect benefits for agents inside the star $S_{1}$. New indirect connections linking the hub of $S_{1}, m_{1}$ to all the players in $S_{1}$ have been created. At the same time, the strength of an indirect connection between two peripheral nodes $i$ and $j$ of $S_{1}$ has been decreased from $\left(\phi(X)+\phi\left(x_{m_{1}}^{i}\right)\right)\left(\phi(X)+\phi\left(x_{m_{1}}^{j}\right)\right)$ to $\phi(X)^{2}$. The difference in indirect benefits can thus be computed as:

$$
\begin{aligned}
\Delta I_{1} & =2\left(s_{1}-1\right) \phi(X)^{2}-\sum_{i, j \in S_{1} \backslash m_{1}} \phi(X)\left(\phi\left(x_{m_{1}}^{i}\right)+\phi\left(x_{m_{1}}^{j}\right)\right)-\sum_{i, j \in S_{1} \backslash m_{1}} \phi\left(x_{m_{1}}^{i}\right) \phi\left(x_{m_{1}}^{j}\right) . \\
& =2\left(s_{1}-1\right) \phi(X)^{2}-2(m-2) \phi(X) \sum_{i \in S_{1} \backslash m_{1}} \phi\left(x_{m_{1}}^{i}\right)-\sum_{i, j \in S_{1} \backslash m_{1}} \phi\left(x_{m_{1}}^{i}\right) \phi\left(x_{m_{1}}^{j}\right) .
\end{aligned}
$$

By convexity of $\phi$,

$$
\sum_{i \in S_{1} \backslash m_{1}} \phi\left(x_{i}^{m_{1}}\right) \leq \phi(X)
$$

and

$$
\sum_{i, j \in S_{1} \backslash m_{1}} \phi\left(x_{m_{1}}^{i}\right) \phi\left(x_{m_{1}}^{j}\right)<\sum_{i \in S_{1} \backslash m_{1}} \phi\left(x_{i}^{m_{1}}\right)^{2} \leq \phi(X)^{2}
$$


so that

$$
\Delta I_{1}>0 .
$$

Hence, aggregate indirect benefits have increased after the merger of the two stars. As the same argument can be repeated with any pair of stars, we have completed step 2 and the proof of the first statement of the Theorem.

Next, suppose that $\phi$ is linear. Let $S^{*}$ be the star with hub at $n$, where all arcs have strength $X+\frac{X}{n-1}$. Let $S$ be any other star with hub $n$ where $s_{i n}$ may not be equal to $s_{j n}$ for $i \neq j$, but where $\sum_{i=1}^{n-1}\left(x_{i}^{n}+x_{n}^{i}\right)=n X$. Of course, total direct benefits are maximized at both $S^{*}$ and $S$. We now show that the sum of indirect benefits in $S^{*}$ is greater than that in $S$.

Without loss of generality let $s_{1 n}$ and $s_{2 n}$ denote the weakest and strongest links in $S$. Consider the effect of increasing investment on $s_{1 n}$ by $\varepsilon$ and simultaneously decreasing investment on $s_{2 n}$ by $\varepsilon$.

The effect on the overall value can be computed as

$$
\Delta V=2\left[\varepsilon\left(s_{2 n}-s_{1 n}\right)-\varepsilon^{2}\right]
$$

Hence, for $\varepsilon$ small enough, $\Delta V>0$ and so local changes in the direction of equalization are profitable. But this implies that the symmetric star has higher value than the asymmetric star.

Several lemmas precede the proof of Theorem 2 .

Lemma 1 Suppose $\phi$ is strictly convex, and $g$ is a connected network which is Nash stable. Then, all agents invest in a single link.

Proof. For any pair of connected agents $(i, j)$, let $W_{i}^{j}$ denote the equilibrium marginal value to $i$ of the connection with $j$ - namely the value of the direct connection to $j$, and of all indirect connections to agents that $i$ accesses through $j$.

Suppose that agent $i$ actually invests on two links to agents $j$ and $k$. The equilibrium values of the connections to $j$ and $k$ are given by

$$
W_{i}=\left(\phi\left(x_{i}^{j}\right)+\phi\left(x_{j}^{i}\right)\right) W_{i}^{j}+\left(\phi\left(x_{i}^{k}\right)+\phi\left(x_{k}^{i}\right)\right) W_{i}^{k} .
$$

Suppose without loss of generality that $W_{i}^{j} \geq W_{i}^{k}$, and consider a deviation, where agent $i$ invests $x_{i}^{j}+x_{i}^{k}$ on the link with agent $j$. This will result in a change in utility

$$
\Delta W_{i} \geq W_{i}^{j} \phi\left(x_{i}^{j}+x_{i}^{k}\right)-W_{i}^{j} \phi\left(x_{i}^{j}\right)-W_{i}^{k} \phi\left(x_{i}^{k}\right)>0,
$$


concluding the proof of the Lemma.

Lemma 2 Suppose $\phi$ is linear, and $g$ is strongly pairwise stable. If there is an agent $i$ who invests in multiple links, then all of $i$ 's neighbors invest fully in the link to $i$.

Proof. For any triple of agents $(i, j, k)$ where $i j, j k \in g$, let $W_{i}^{j, k}$ denote the equilibrium marginal value to $i$ of the connection to $k$ through $j$.

Suppose that agent $i$ invests on two links to agents $j$ and $k$. There are two cases to consider.

Case 1: Both $j$ and $k$ invest on two or more links, so that $x_{j}^{i}<X$ and $x_{k}^{i}<X$.

Assume that $s_{i j} \leq s_{i k}$. We construct a joint reallocation of resources for players $i$ and $j$ which makes both players strictly better off. Consider first a reallocation, where agent $i$ shifts $\varepsilon$ resources from the link to $k$ to the link to $j$. Because $\phi$ is linear and player $i$ invests in both links, the marginal values of the connections to $j$ and $k$ must be equal, that is $W_{i}^{j}=W_{i}^{k}$. Hence, by reallocating investment to the link with $j$, agent $i$ 's utility cannot decrease. Consider now the utility to agent $j$ of the connection to $i$ :

$$
W_{j}=s_{i j}\left(1+s_{i k} W_{j}^{i, k}+\sum_{m \neq j, k} s_{i m} W_{j}^{i, m}\right) .
$$

Hence, the reallocation of investment will result in a utility change

$$
\Delta W_{j}=\varepsilon\left(1+\sum_{m \neq j, k} s_{i l} W_{j}^{i, m}+W_{j}^{i, k}\left(s_{i k}-s_{i j}\right)\right)-\varepsilon^{2} W_{j}^{i, k} .
$$

For $\varepsilon$ close to zero, the change in utility is positive as $s_{i j} \leq s_{i k}$.

Next, consider an agent $l$ such that $x_{j}^{l}>0$ and consider a reallocation where agent $j$ shifts $\delta$ resources from the link to $l$ to the link to $i$. Because $x_{j}^{i}$ may be equal to zero, this reallocation may result in a utility loss for agent $j$. However, this utility loss must be continuous in $\delta$ and hence, for any $\varepsilon$, one can find $\delta(\varepsilon)$, such that the total effect of the reallocation of resources $(\delta, \varepsilon)$ on the utility of $j$ is strictly positive. Now consider agent $i$. Since agent $i$ could have chosen to connect directly to agent $l$, the equilibrium marginal value of $i$ 's connection to $j$ must be at least as large as the marginal value of a possible direct connection to $l$. So,

$$
W_{i}^{j}=1+s_{j l} W_{i}^{j, l}+\sum_{m \neq i, l} s_{j m} W_{i}^{j, m} \geq W_{i}^{j, l} .
$$


Now the effect of the reallocation of resources $\delta$ on the utility of agent $i$ is given by:

$$
\Delta W_{i}=\delta\left(1+\sum_{m \neq i, l} s_{j m} W_{i}^{j, m}+W_{i}^{j, l}\left(s_{j l}-s_{i j}\right)\right)-\delta^{2} W_{i}^{j, l} .
$$

Using equation 4 ,

$$
\Delta W_{i} \geq \delta\left(1-s_{i j}\right) W_{i}^{j, l}-\delta^{2} W_{i}^{j, l},
$$

which is strictly positive for $\delta$ close to zero. Hence, we have constructed a joint reallocation of resources which makes both players $i$ and $j$ strictly better off.

Case 2: Suppose $x_{j}^{i}=X$, but $x_{k}^{i}<X$.

From the proof above, it is clear that if $s_{i j} \geq s_{i k}$, then individuals $i$ and $k$ can jointly plan a profitable deviation. So, assume that $s_{i j}<s_{i k}$. Since $x_{j}^{i}=X$, this means that $X>x_{k}^{i}>0$. Let $x_{k}^{l}>0$ for some $l$. Since $i$ invests on $k, W_{i}^{k} \geq W_{i}^{l}$. Similarly, since $k$ invests on $i, W_{k}^{i} \geq W_{k}^{j}$. Suppose $k$ transfers some resource from the link to $l$ to the link to $i$. Then, $i$ 's total utility strictly increases since he gets the additional direct benefit, and there cannot be any loss in indirect benefit since $W_{i}^{k} \geq W_{i}^{l}$. Moreover, $k$ is indifferent since $W_{k}^{i}=W_{k}^{i}$. For an exactly analogous reason, a transfer of resource by $i$ from the link to $j$ to the link to $k$ makes $k$ better off and leaves $i$ indifferent. So, $i$ and $k$ have a profitable joint deviation.

Lemma 3 Let $g$ be a connected network which is not a tree. Then,

(i) If $\phi$ is strictly convex, then $g$ is not Nash stable.

(ii) If $\phi$ is linear, then $g$ is not strongly pairwise stable.

Proof. Let $g$ contain a cycle $\{(12),(23), \ldots,(r-1, r),(r 1)\}$. Denote $R=$ $\{1,2, \ldots, r\}$.

(i) Since $\phi$ is strictly convex, Lemma 1 implies that all agents invest only on one link. For each $i \in R$, let $L_{i}$ denote the set of nodes contained in $N \backslash R$ which can be accessed through node $i$. Note that each pair $L_{i}, L_{j}$ must be disjoint sets since agents invest in only one link, there can be at most one cycle.

Let $V_{i}$ be the total benefit that $i$ gets from nodes in $L_{i}$. We first show that $V_{i} \equiv V$ for all $i \in R$.

Suppose not, so that $V_{i} \neq V_{j}$ for some $i, j \in R$. Then, there must be a pair of adjacent nodes $i, i+1$ such that $V_{i+1}>V_{i}$. Then, $i-1$ is better off connecting to $i+1$ rather than to $i$. Hence, $V_{i} \equiv V$ for all $i \in R$.

So, $i$ gets a benefit of $\phi(X)^{k}$ from node $i+k$ in $R$ and $\phi(X)^{k} V$ from nodes in $L_{i+k}$. Let $B=1+V$. Then, if $r$ is odd, agent $i$ initially obtains

$$
U_{i}=2 B \sum_{k=1}^{(r-1) / 2} \phi(X)^{k}
$$


If $r$ is even, then $i$ obtains

$$
U_{i}=2 B \sum_{k=1}^{(r-2) / 2} \phi(X)^{k}+\phi(X)^{r / 2} B
$$

Now suppose that agent $i$ in the cycle unilaterally deviates and chooses to invest $X$ on the link with $i-1$. This increases the strength of the link $(i-1, i)$ to $2 \phi(X)$. Then, after the deviation, she receives

$$
U_{i}^{\prime}=2 B \sum_{k=1}^{(r-1)} \phi(X)^{k}
$$

Hence, irrespective of whether $r$ is odd or even, this deviation makes agent $i$ strictly better off.

(ii) We first prove that if $\phi$ is linear and $g$ is strongly pairwise stable, then all agents in the cycle invest on only one link.

Suppose in contradiction that an agent $i$ in the cycle invests on multiple links. From Lemma 2, both agents $i-1$ and $i+1$ must invest fully on the link with $i$. Let $k$ be the smallest integer such that $i-k$ invests both on $i-k+1$ and $i-k-1$. Such $k$ must exist to complete the cycle. Then, lemma 2 requires that $i-k+1$ invests fully on the link with $i-k$. But, by assumption $i-k+1$ invests fully on the link with $i-k+2$.

Now, take any agent $j$ in $L_{i}$ who is connected to $i$, and $k \in L_{i+1}$ who is connected to $i+1$. Lemma 2 implies that $j$ invests fully on the link with $i$ and $k$ invests fully on the link with $i+1$. Repeated application of lemma 2 implies that all agents in $L_{i}$ and $L_{i+1}$ in fact invest only on one link.

The rest of the proof is identical to that of (i) above. ${ }^{16}$

Proof of Theorem 2: From lemma 3, we know that only trees can be Nash stable if $\phi$ is strictly convex or strongly pairwise stable if $\phi$ is linear.

Now, consider a tree of diameter greater than or equal to 3 . Let $i$ and $j$ be two terminal nodes at a distance greater than or equal to 3 , and let $k$ and $l$ be their predecessors in the tree (where $k \neq l$ as the distance between $i$ and $j$ is greater than or equal to 3 ). Agent $i$ could have chosen to invest his endowment on player $l$ instead of $k$, so that

$$
W_{i}^{k} \geq W_{i}^{l}=W_{j}^{l}+R(l, j)-R(l, i),
$$

\footnotetext{
${ }^{16}$ Notice that in the proof of (i) we have used strict convexity of $\phi$ only to conclude that all agents invest only on one link.
} 
where the last equality results from the fact that by investing on $l$, agent $i$ would have received the same marginal value as $j$, but would have in addition gained a connection of length 2 to $j$ and lost the indirect connection between $j$ and $i$. By a similar computation,

$$
W_{j}^{l} \geq W_{i}^{k}+R(k, i)-R(k, j) .
$$

Summing up these inequalities, we obtain: $R(l, i)+R(k, j) \geq R(k, i)+R(l, j)$, a contradiction since $R(l, i)=R(k, i) R(k, l)$ and $R(k, j)=R(l, j) R(k, l)$ with $R(k, l)<1$.

This shows that if $g$ is not a collection of stars, then it cannot be Nash stable if $\phi$ is strictly convex or strongly pairwise stable if $\phi$ is linear.

Suppose that the network contains two stars, $S_{1}$ and $S_{2}$, with $\left|S_{1}\right|=s_{1} \geq s_{2}=$ $\left|S_{2}\right|$. If $\phi$ is strictly convex, then from lemma 1 , the hub of the star must invest fully on one link. Consider then a unilateral deviation from a peripheral agent $i$ of $S_{2}$ in which the hub does not invest. By connecting to the hub of $S_{1}$, agent $i$ obtains a payoff $V_{i}^{\prime}=\phi(X)+\left(s_{1}-2\right) \phi(X)^{2}+2 \phi(X) 2=\phi(X)+s_{1} \phi(X)^{2}$ whereas she initially received a payoff of $V_{i}=\phi(X)+\left(s_{2}-3\right) \phi(X)^{2}+2 \phi(X)^{2}=\phi(X)+\left(s_{2}-1\right) \phi(X)^{2}$. Since $V_{i}<V_{i}^{\prime}$, this shows that a network containing two or more stars cannot be Nash stable. It is easy to check that a star in which the hub invests fully on one link is Nash stable. This concludes the proof of part (i) of the theorem.

If $\phi$ is linear, consider the peripheral agent $i$ who receives the lowest benefit. Without loss of generality, suppose that she belongs to star $S_{2}$ and let $i_{2}^{*}$ denote the hub of star $S_{2}$. and $i_{1}^{*}$ the hub of star $S_{1}$. Consider the following joint deviation by agents $i$ and $i_{1}^{*}$ : agent $i$ connects fully to $i_{1}^{*}, x_{i}^{i_{1}^{*}}=X$; agent $i_{1}^{*}$ shifts away investment $x_{i_{1}^{*}}^{j}$ from some peripheral agent $j$ in start $S_{1}$ and invests this amount in the link to $i$. After this deviation, agent $i_{1}^{*}$ 's payoff has increased from $s_{1} X$ to $\left(s_{1}+1\right) X$ and agent $i$ 's payoff has increased from $V_{i}$ to $V_{j}+X s_{i i_{1}^{*}}>V_{i}$. This shows that if $\phi$ is linear, then a strongly pairwise network must be a connected star.

Suppose now that

$$
X<\frac{(n-1)^{2}}{n\left(n^{2}-3 n+3\right)}
$$

We want to show that no star can be strongly pairwise stable. Let $g$ be any connected star with the hub being individual 1, and suppose that $i$ and $j$ are the two peripheral agents with lowest utility. This implies that $x_{1}^{i} x_{1}^{j} \leq\left(\frac{X}{n-1}\right)^{2}$ since the hub's investment on $i$ and $j$ cannot exceed $2 X /(n-1)$.

Consider a joint deviation in which players $i$ and $j$ invest fully on the link $i j$. Then, the gain in direct benefit for each of these two players is $X$. Each of these 
players loses an indirect benefit of $X\left(X+x_{1}^{k}\right)$ from each player $k \neq 1, i, j$ and the indirect benefit of $\left(X+x_{1}^{i}\right)\left(X+x_{1}^{j}\right)$. So, the total loss in indirect benefit to player $l, l=i, j$ is

$$
\begin{aligned}
L & =X \sum_{k \neq 1, i, j}\left(X+x_{1}^{k}\right)+\left(X+x_{1}^{i}\right)\left(X+x_{1}^{j}\right) \\
& =(n-1)^{2} X^{2}+x_{1}^{i} x_{1}^{j} \\
& \leq(n-1)^{2} X^{2}+\frac{X^{2}}{(n-1)^{2}} \\
& <X
\end{aligned}
$$

where the last inequality follows from equation 5 . This shows that if the inequality in equation 5 holds, then the set of strongly pairwise networks is empty.

Conversely, consider the connected star in which the hub invests equally on each link, and suppose that $X \geq \frac{(n-1)^{2}}{n\left(n^{2}-3 n+3\right)}$. As the hub of the star gets the maximal payoff, $n X$, she never has any incentive to deviate. No peripheral agent has any unilateral profitable deviation. Now, consider a joint deviation by a pair of peripheral agents $i, j$. The only possible such deviation is for player $i$ to shift some resource from her investment on the link with the hub to a link with player $j$, and for player $j$ to reciprocate. Given the linearity of $\phi$, if this deviation is jointly profitable for transfers $\left(\delta_{i}, \delta_{j}\right)$, then it is profitable when $i$ and $j$ invest fully on their own link. Then, the gain in direct benefit is $X$. The loss in indirect benefit is exactly $(n-1)^{2} X^{2}+\frac{X^{2}}{(n-1)^{2}} \leq X$. Hence, the connected star in which the hub invests an equal amount on each link is strongly pairwise stable. This completes the proof of the theorem.

\section{Proof of Theorem 3:}

(i) The proof follows the same lines as the proof of Theorem 1.

Step 1: Observe first that we can restrict attention to trees. If a network contains a cycle, then consider the weakest link on the cycle, and reallocate the investments over the other links. Since the weakest link in the cycle was never used on any path (or agents were indifferent between using it or an alternative path), this reallocation must weakly increase the aggregate benefits in the network. Consider now any tree $h$ on $m$ nodes, with link strengths $\left\{z_{1}, \ldots, z_{m-1}\right\}$, and the star $S$ corresponding to $h$ as in the proof of Theorem 1. Denote the link strengths in $S$ as $\left\{s_{1}, \ldots, s_{m-1}\right\}$. Direct benefits are exactly identical in $S$ and in $h$. The sum of indirect benefits in the star $S$ is easily calculated.

$$
I=2 \sum_{i=1}^{m-2}(m-i-1) s_{m-i}
$$


To check this, note that the link $s_{m-1}$ is the minimum in all comparisons, and so the sum of indirect benefits obtained between $m-1$ and other nodes is $2(m-2) s_{m-1}$. Similarly, $s_{m-2}$ is the minimum in $(m-3)$ comparisons and so on.

Now, consider indirect benefits in $h$. Note that the arc $z_{m-1}$ must also be involved in at least $(m-2)$ comparisons in the graph $h$ (the minimum being attained if $z_{m-1}$ connects some terminal node). Similarly, for any $t$, the $t \operatorname{arcs}$ with the lowest values, $\left\{z_{m-t}, \ldots, z_{m-1}\right\}$ must be the minimum in at least $(m-$ $2)+(m-3)+\ldots+(m-t-1)$ connections (the minimum being attained if they both connect to some terminal node). This establishes that the sum of direct and indirect benefits is at least as high in $S$ as in $h$.

Step 2: We now show that if the graph contains two stars $S_{1}$ and $S_{2}$, it is dominated by the graph where the two stars are merged into a single star, as in the proof of Theorem 1. By merging the two stars into a single star with hub $m_{2}$, direct benefits have increased. Furthermore, indirect benefits for players in star $S_{2}$ have strictly increased. For a peripheral agent $i$ in star $S_{1}$, indirect benefits were equal to

$$
\begin{aligned}
I_{i} & =\sum_{j \in S_{1} \backslash m_{1}} \phi(X)+\min \left\{\phi\left(x_{m_{1}}^{i}\right), \phi\left(x_{m_{1}}^{j}\right)\right\} \\
& =\left(m_{1}-1\right) \phi(X)+\sum_{j \in S_{1} \backslash m_{1}} \min \left\{\phi\left(x_{m_{1}}^{i}\right), \phi\left(x_{m_{1}}^{j}\right)\right\} .
\end{aligned}
$$

In the new star, indirect benefits are given by:

$$
I_{i}^{*}=\left(m_{1}+m_{2}-1\right) \phi(X) .
$$

As $m_{2} \geq 1$ and $\phi(X) \geq \sum_{j \in S_{1} \backslash m_{1}} \min \left\{\phi\left(x_{m_{1}}^{i}\right), \phi\left(x_{m_{1}}^{j}\right)\right\} I_{i}^{*} \geq I_{i}$ and indirect benefits cannot have decreased.

This concludes the proof of part (i).

(ii) Next suppose that $\phi$ is linear, and that the hub invests different amounts on its links with peripheral nodes. Let $i$ be a node for which $x_{n}^{i}$ is maximal, and $j$ a node for which $x_{n}^{j}$ is minimal. Consider a reallocation of investments, $\widetilde{x_{n}^{i}}=x_{n}^{i}-\varepsilon$, $\widetilde{x_{n}^{j}}=x_{n}^{j}+\varepsilon$. This reallocation does not affect direct benefits. For $\varepsilon$ small enough, it only reduces indirect benefits between $i$ and other players who are connected to the hub by links of maximal strength by $\varepsilon$. Indirect benefits between player $j$ and all these players have been increased by $\varepsilon$, and indirect benefits between players $i$ and $j$ have strictly increased. Hence, this reallocation of investments has strictly increased the value of the graph, showing that the hub must put equal weight on all links with peripheral agents. 
Now, in the symmetric star, each link has strength $\left(X+\frac{X}{n-1}\right)$. Since there are $n-1$ links, each agent gets a benefit of $n X$. Since this equals the total resource available, the symmetric star must be strongly pairwise stable.

(iii) To show that, if $\phi$ is strictly convex, the star where the hub invests on a single link is strongly pairwise stable, notice that the hub and the peripheral agent in which he invests obtain the maximal benefit of $n \phi(X)$ and have no incentive to move.

Other peripheral agents have a benefit of $(n-1) \phi(X)$. If two peripheral agents move, they have no incentive to reallocate their links in such a way that they form a cycle. Hence, either they reallocate all their investments on their link and they obtain $2 \phi(X)$ which is smaller than their initial payoff. Or one of them reallocates his investment from the hub to the peripheral agent, resulting in the same payoff of $(n-1) \phi(X)$ as before. Hence, there does not exist any profitable deviation.

Proof of Theorem 4: (i) Suppose $g$ is efficient and has a component with three or more nodes, where two nodes have degree one. Denote these nodes by $i$ and $j$ and their immediate predecessors by $k$ and $l$ respectively. Because the component is connected, the degrees of $k$ and $l$ are necessarily greater than one. But this implies that $x_{k}^{i}<X$ and $x_{l}^{j}<X$. Furthermore because $\sum_{m \in N \backslash\{i\}} x_{k}^{m} \leq X, x_{k}^{m} \leq X-x_{k}^{i}$ for all node $m \neq i$ to which $k$ is connected. Now, this implies that the value of the indirect connection between $i$ and $j$ in the graph is strictly smaller than $\min \left\{X-x_{k}^{i}, X-x_{l}^{j}\right\}$. Furthermore, in an efficient graph, $x_{i}^{k}=x_{k}^{i}$ and $x_{j}^{l}=x_{l}^{j}$ so that individual $i$ can invest $X-x_{k}^{i}$ in the direct link with $j$ and individual $j$ can invest $X-x_{l}^{j}$ in the link with $i$. But, because the value of the indirect link is smaller than $\min \left\{X-x_{k}^{i}, X-x_{l}^{j}\right\}$, the investment in the direct link strictly increases the value of the graph, yielding a contradiction. This completes the proof of part (i).

(ii) To show that the symmetric circle is efficient for low values of $n$, consider the different values of $n$ in turn. For $n=3$, the circle is the only connected graph which is not a tree. Now, notice that direct benefits are equal to $n X$ and hence are maximal in the circle. For $n=4,5$, we show that the circle also maximizes the value of indirect benefits. Notice first that the value of an indirect connection is always bounded above by $(X / 2)^{2}$ as the middle player must allocate $X$ over at least two links. For $n=4$ and $n=5$ all indirect connections in the circle are of length 2 and have value $(X / 2)^{2}$. Hence, the circle achieves the highest sum of indirect links and is efficient. It is easy to check that any other allocation of investments results in a lower value of indirect links, so the circle with links of 
equal strength is uniquely efficient.

Suppose now that $n=6,7$. The indirect benefit for any node in the circle is

$$
I=\frac{X^{2}}{2}+\frac{X^{3}}{4}
$$

Consider any other graph $g$. If this graph is to "dominate" the cycle, then at least one node (say $i^{*}$ ) has to derive an indirect benefit exceeding $I$. For each $k$ , check that the circle maximizes indirect benefits from nodes at a distance of $k$. So, if $i$ is to derive a larger indirect benefit in $g$, it must have more than two nodes at a distance of $2 .{ }^{17}$

It is tedious to show that the maximum indirect benefit that $i^{*}$ can derive occurs when $i^{*}$ has two neighbors, $j_{1}, j_{2}$, with each neighbor of $i^{*}$ having three neighbors including $i^{*}$ itself. Moreover, the optimum pattern of allocation from the point of view of $i^{*}$ is

$$
x_{i}^{j_{1}}=x_{i}^{j_{2}}=x_{j_{2}}^{i}=x_{j_{2}}^{i}=\frac{1}{2}
$$

This yields $i^{*}$ a total indirect benefit of $\frac{X^{2}}{2}<I$. This completes the proof of part (ii).

(iii) Finally, we show that the symmetric circle is strongly pairwise stable. In the symmetric cycle, each $i$ gets a direct benefit of $X$. No pattern of investment can result in higher direct benefits. So, we check whether a deviation by $i$ and $j$ can improve their indirect benefits.

Suppose $i$ and $j$ are neighbors in the cycle. Consider the effect on $i$ of increasing investment to $\frac{X}{2}+y$ by both $i$ and $j$ on the link $i j$, and decreasing their investments on their other neighbors by $y$. The change in indirect benefit for $i$ from $j$ 's other neighbor is $\left(\frac{X}{2}+y\right)\left(\frac{X}{2}-y\right)-\left(\frac{X}{2}\right)^{2}<0$. A similar calculation shows that $i$ also loses from nodes which are further away.

Suppose $i$ and $j$ are not neighbors in the cycle. Let $i$ and $j$ mutually invest $y$ each on the link $i j$ and simultaneously decrease investment on their previous neighbors by $\frac{y}{2}$. It is easy to check that this is the best possible deviation.

Clearly, this can only increase indirect benefit for $i$ if there is some $k$ such that the distance between $i$ and $k$ is now lower. This means that $i$ accesses $k$ through $j$. Let $k$ be a neighbor of $j$. Then, the indirect benefit for $i$ from $k$ is

$$
I=y\left(\frac{X-y}{2}\right)=\frac{X y}{2}-\frac{y^{2}}{2}
$$

\footnotetext{
${ }^{17}$ Since $n \leq 7$, the maximum distance between any two nodes in the circle is 3 .
} 
Now, $i$ has reduced the strength of links with each of its previous neighbors by $\frac{y}{2}$. Also, since $k$ is not at a distance of 2 from $i$ in the cycle, there must be some node $m$, distinct from $k$ which is at a distance of 2 from $i$. The loss in indirect benefit for $i$ from $m$ is

$$
I^{\prime}=\left(\frac{X-y}{2}\right) \frac{X}{2}-\frac{X^{2}}{4}=\frac{X y}{2}
$$

Hence, the indirect benefit for $i$ from $k$ is lower than the loss in indirect benefit from $m$.

Repeating this argument, it can be shown that $i$ 's total indirect benefit will actually go down as a result of the deviation. 